\title{
A multi-center clinical investigation on invasive Streptococcus pyogenes infection in China, 2010-2017
}

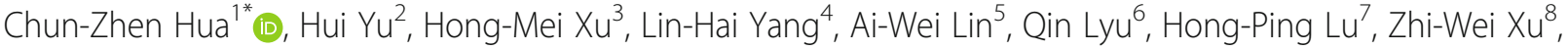 \\ Wei Gao ${ }^{9}$, Xue-jun Chen ${ }^{10}$, Chuan-Qing Wang ${ }^{11}$ and Chun-mei Jing ${ }^{12}$
}

\begin{abstract}
Background: Invasive S. pyogenes diseases are uncommon, serious infections with high case fatality rates (CFR). There are few publications on this subject in the field of pediatrics. This study aimed at characterizing clinical and laboratory aspects of this disease in Chinese children.

Patients and methods: A retrospective study was conducted and pediatric in-patients with S. pyogenes infection identified by cultures from normally sterile sites were included, who were diagnosed and treated in 9 tertiary hospitals during 2010-2017.

Results: A total of 66 cases were identified, in which 37 (56.1\%) were male. The median age of these patients, including 11 neonates, was 3.0 y. Fifty-nine (89.4\%) isolates were determined from blood. Fever was the major symptom $(60 / 66,90.9 \%)$ and sepsis was the most frequent presentation $(64 / 66,97.0 \%$, including $42.4 \%$ with skin or soft tissue infections and $25.8 \%$ with pneumonia. The mean duration of the chief complaint was (3.8 \pm 3.2$) \mathrm{d}$. Only 18 (27.3\%) patients had been given antibiotics prior to the hospitalization. Among all patients, 15 (22.7\%) developed streptococcal toxin shock syndrome (STSS). No S. pyogenes strain was resistant to penicillin, ceftriaxone, or vancomycin, while $88.9 \%(56 / 63)$ and $81.4 \%(48 / 59)$ of the tested isolates were resistant to clindamycin and erythromycin respectively. Most of the patients were treated with $\beta$-lactams antibiotics and $36.4 \%$ had been treated with meropenem or imipenem. Thirteen (19.7\%) cases died from infection, in which 9 (13.6\%) had complication with STSS.
\end{abstract}

Conclusions: Invasive S. pyogenes infections often developed from skin or soft tissue infection and STSS was the main cause of death in Chinese children. Ongoing surveillance is required to gain a greater understanding of this disease.

Keywords: Streptococcus pyogenes, invasive infection, Group a streptococcus, Children, Streptococcal toxin shock syndrome

\section{Background}

Group A streptococcus (GAS), such as Streptococcus pyogenes (S. pyogenes), is a major human pathogen and causes a wide range of diseases, from mild skin and soft tissue infections, pharyngitis, tonsillitis to severe invasive diseases in humans [1]. A case of invasive GAS (iGAS) disease is defined as GAS is isolated from a normally

\footnotetext{
* Correspondence: huachunzhen@zju.edu.cn

'Division of Infectious Diseases, The Children's Hospital, Zhejiang University

School of Medicine, Hangzhou 310003, People's Republic of China

Full list of author information is available at the end of the article
}

sterile body site or from a wound specimen obtained from a patient with necrotizing fasciitis or streptococcal toxic shock syndrome (STSS) [2]. Epidemiology of iGAS infection is highly variable in different population and the annual incidences varied from $2.5 / 100,000$ to $101 /$ 100,000 in children around the world [2-5]. The iGAS disease causes serve infection associated with high mortality, especially in those who developed STSS [1-5]. Nonetheless, no active surveillance on iGAS infections was conducted and data regarding clinical characteristics and risk factors of such pediatric patients are limited in

(c) The Author(s). 2019 Open Access This article is distributed under the terms of the Creative Commons Attribution 4.0 International License (http://creativecommons.org/licenses/by/4.0/), which permits unrestricted use, distribution, and reproduction in any medium, provided you give appropriate credit to the original author(s) and the source, provide a link to the Creative Commons license, and indicate if changes were made. The Creative Commons Public Domain Dedication waiver (http://creativecommons.org/publicdomain/zero/1.0/) applies to the data made available in this article, unless otherwise stated. 
China. Though S. pyogenes, the most common member of GAS, was rarely isolated from normally sterile site, it was an important pathogen due to causing life-threaten infection which were associated with hospital-patient disputes in China. The aim of this work is to improve the understanding of the clinical and laboratory characteristics and treatment of invasive $S$. pyogenes disease (ISPD) in children at a regional level.

\section{Methods}

\section{Setting and patients}

Retrospectively, patients with at least one positive culture from normally sterile body sites for S. pyogenes were included at 9 tertiary hospitals in 9 cities in China. Every institution had 300-1500 pediatric beds and approximately 10,000-39,000 hospitalizations each year. The patients who were admitted during the years 2010-2017 and with a disease onset at $\leq 18$ years of age were included in the study. Patients' demographics, type of infection, clinical presentation, treatment, microbiological data, hospital stay and evolution were collected from electronic medical records.

\section{Identification of organism and drug-susceptibility}

Each organism was identified using the Vitek system GPI card (Mérieux, France) or Maldi-TOF mass spectrometry analysis (Bruck, Germany). Antimicrobial susceptibility test was performed using Kirby-Bauer method according to the criteria of the Clinical and Laboratory Standards Institute at the 9 institutions. Repeated S. pyogenes isolation from different samples of the same patient was considered as one $S$. pyogenes clone.

\section{Statistical analysis}

The demography of the patients was summarized by mean, standard deviation (SD), interquartile range and proportion as appropriate.

\section{Ethics}

This study was performed under the institutions opt-out passive consent policy and approved by the ethics committee and the Institutional Board of Privacy and Security at the hospitals (2018-IEC-047). Patients and guardians wishing to withdraw from the study were able to contact the principal investigator through information provided on notifications publically posted by the institution's ethics committee.

\section{Results}

\section{Patients' baseline characteristics}

A total of 66 hospitalized patients with culture-confirmed ISPD were included and 37 (56.31\%) were male. Age of the patients ranged from $2 \mathrm{~h}$ to $15 \mathrm{y}$ and the median age was 3.0 y (p25-75: 0.48-8 y). Eleven (16.7\%) were neonate. Thirteen (19.7\%) had underlying condition. Forty-eight (72.7\%) had not been prescribed with any antibiotics before specimen were collected for culture. Patients' underlying condition and prior antibiotics use were summarized in Table 1.

\section{Clinical characteristics}

Of all 66 patients, disease course was $3.8 \pm 3.2 \mathrm{~d}(2 \mathrm{~h}-20$ d). Sixty (90.9\%) patients had fever with mean temperature as $39.4^{\circ} \pm 0.9^{\circ} \mathrm{C}$. Of the other six patients who did not have fever, $4(6.1 \%)$ were neonates and $2(3.0 \%)$ were infants at 1 month of age. Most frequent clinical manifestation was sepsis (64 cases, $97.0 \%), 59$ of which were blood culture confirmed. Fifteen (22.7\%) developed STSS. One patient had been misdiagnosed as Kawasaki disease because of rash, conjunctival congestion, strawberry tongue, cervical lymphadenopathy and peeling around the anus. Lumbar puncture was performed in $23(34.8 \%)$ patients and 8 (12.1\%) were identified as meningitis by laboratory examination of cerebrospinal fluid, though only 2 of the 8 had a positive result as $S$. pyogenes by culture. One patient had concomitant infection with both S. pyogenes and Streptococcus pneumonia in blood stream. Nineteen $(28.8 \%)$ patients had multi-organ failure. The clinical manifestation was shown in Table 2.

Laboratory results (1) Blood Routine Initial leukocyte count was quite variable. Leukopenia was presented in 7 (10.6\%) patients and leukocytosis was presented in 44 (66.7\%) patients. Fifty-nine (89.4\%) patients had CRP levels higher than normal value $(10 \mathrm{mg} / \mathrm{L})$. Procalcitonin levels in sera were detected in 40 patients and 33 (82.5\%) had levels higher than normal value $(0.5 \mathrm{mg} / \mathrm{L})$. The blood routine, $\mathrm{C}$ reaction protein levels, and procalcitonin levels in the 66 patients were shown in Table 3. (2) Isolates and antibiotic-resistance Sixty-six strains of S. pyogenes were identified in 66 patients. The vast majority of isolates, 59 (89.4\%), were obtained from blood, with the remaining from cerebrospinal fluid (2, 3.0\%), joints $(2,3.0 \%)$, pleural fluid $(2,3.0 \%)$ and peritoneal fluid $(1,1.5 \%)$. S. pyogenes was isolated from multiple sites for some patients, such as 3 (4.5\%) patients from both blood and pyogenic fluids from skin and soft tissue, two (3.0\%) patients from both blood and purulent exudation from tonsil, and 2 (3.0\%) from both blood and articular fluid. All tested strains were phenotypically sensitive to penicillin, ceftriaxone, cefotaxime, vancomycin, and lynezolid. The resistant rate to erythromycin, tetracycline, clindamycin and trimethoprim-sulfamethoxazole were 88.9, 81.5, 81.4 and $72.0 \%$ respectively. Table 4 showed the resistant rates of $S$. pyogenes strains against 10 antibiotics during 2010-2017 in the 9 tertiary hospitals. 
Table 1 The underlying condition and prior antibiotics use in the 66 patients with invasive S. pyogenes infection

\begin{tabular}{|c|c|c|c|c|c|}
\hline Underlying condition & $\mathrm{N}$ & $\%$ & Previous antibiotics & $\mathrm{N}$ & $\%$ \\
\hline Leukemia & 2 & 3.0 & Azithromycin, IV $\times 2-5 \mathrm{~d}$ & 7 & $\overline{10.6}$ \\
\hline Varicella & 2 & 3.0 & Cephalosporins, Oral $\times 3-5 d$ & 3 & 4.6 \\
\hline Skin burn & 2 & 3.0 & Azithromycin, Oral $\times 3 \mathrm{~d}$ & 1 & 1.5 \\
\hline Skin hemangioma & 2 & 3.0 & Erythromycin, Oral $\times 3 \mathrm{~d}$ & 1 & 1.5 \\
\hline Nephrotic syndrome & 1 & 1.5 & Piperacillin-tazobactam, IV × $\mathrm{d}$ & 1 & 1.5 \\
\hline \multirow[t]{2}{*}{ Still's disease } & 1 & 1.5 & Erythromycin, IV $\times 1 \mathrm{~d}$; & 1 & 1.5 \\
\hline & & & Clindamycin, $\mathrm{IV} \times 1 \mathrm{~d}$ & & \\
\hline \multirow[t]{2}{*}{ Cerebral trauma } & 1 & 1.5 & Azithromycin, IV $\times 5 \mathrm{~d}$; & 1 & 1.5 \\
\hline & & & Clindamycin, IV $\times 1 \mathrm{~d}$ & & \\
\hline \multirow[t]{2}{*}{ Craniocerebral trauma } & 1 & 1.5 & Meropenem + vancomycin + & 1 & 1.5 \\
\hline & & & Metronidazole, IV $\times 3 \mathrm{~d}$ & & \\
\hline \multirow[t]{2}{*}{ Cerebrospinal fluid rhinorrhea } & 1 & 1.5 & Meropenem, IV $\times 3 \mathrm{~d}$; & 1 & 1.5 \\
\hline & & & Lynezoliane, IV× $1 \mathrm{~d}$ & & \\
\hline \multirow[t]{4}{*}{ None } & 53 & 80.5 & Piperacillin-tazobactam, IV $\times 1 \mathrm{~d}$; & 1 & 1.5 \\
\hline & & & Clindamycin, IV $\times 4$ d; & 1 & 1.5 \\
\hline & & & Vancomycin + Mezlocillin, IV ×1d & & \\
\hline & & & None & 47 & 71.3 \\
\hline Total & 66 & 100 & Total & 66 & 100 \\
\hline
\end{tabular}

Note: IV, intravenous

Table 2 The clinical manifestation and antibiotics for therapy in the 66 patients with invasive S. pyogenes infection

\begin{tabular}{|c|c|c|c|c|c|}
\hline Clinical manifestation & $\mathrm{N}$ & $\%$ & Treatment with antibiotics & N & $\%$ \\
\hline Meningitis with cerebral trauma & 1 & 1.5 & ${ }^{a}$ Penicillins or/ and Cephalosporins & 27 & 40.9 \\
\hline Pneumonia with pleural effusion & 1 & 1.5 & Carbapenems + vancomycin & 7 & 10.6 \\
\hline Sepsis (64 cases, 97.0\%) & & & Carbapenems, penicillin & 3 & 4.6 \\
\hline Skin / soft tissue infections & 21 & 31.8 & Vancomycin + cephalosporins, Cephalosporins & 3 & 4.6 \\
\hline Severe pneumonia ( 4 with pleural effusion) & 10 & 15.2 & Vancomycin & 2 & 3.0 \\
\hline Suppurative arthritis and osteomyelitis & 6 & 9.1 & Carbapenems & 2 & 3.0 \\
\hline Both Pneumonia and skin / soft tissue infections (1 with pleural effusion) & 6 & 9.1 & Carbapenems, Cephalosporins & 2 & 3.0 \\
\hline Suppurative tonsillitis & 3 & 4.5 & Vancomycin + cephalosporins & 2 & 3.0 \\
\hline Meningitis & 3 & 4.5 & Carbapenems + vancomycin, vancomycin & 2 & 3.0 \\
\hline $\begin{array}{l}\text { Skin / soft tissue infections, suppurative arthritis, osteomyelitis and } \\
\text { necrotising fasciitis }\end{array}$ & 2 & 3.0 & $\begin{array}{l}\text { Carbapenems + vancomycin, Vancomycin }+ \\
\text { cephalosporins }\end{array}$ & 2 & 3.0 \\
\hline Both pneumonia and meningitis & 1 & 1.5 & Vancomycin + cephalosporins, Penicillin & 2 & 3.0 \\
\hline Both skin / soft tissue infections and meningitis & 1 & 1.5 & ${ }^{\mathrm{b}}$ Other regimen & 10 & 15.3 \\
\hline Both mastoiditis and otitis media & 1 & 1.5 & No antibiotics & 2 & 3.0 \\
\hline Without identified focus & 10 & 15.3 & & & \\
\hline Total & 66 & 100 & Total & 66 & 100 \\
\hline
\end{tabular}

aenicillins (4 cases); Cephalosporins (13 cases); Penicillins + Cephalosporins (5 cases); Cephalosporins, penicillins (5 cases)

${ }^{\mathrm{b}}$ Clindamycin, azithromycin (1 case); azithromycin, vancomycin (1 case); Carbapenems+ linezolid, penicillin + linezolid (1 case); Carbapenems + vancomycin, lynezolid (1 case); Carbapenems + vancomycin, penicillin + lynezolid (1 case); Carbapenems + vancomycin, cephalosporins (1 case); Carbapenems, lynezolid ( case); Carbapenems + vancomycin, vancomycin, cephalosporins (1 case); Vancomycin + cephalosporins, penicillin (1 case); Vancomycin + penicillin, penicillin (1 case) 
Table 3 The Leukocyte count, Neutrophils count, C reaction protein levels, and procalcitonin levels in the 66 patients with invasive S. pyogenes infection

\begin{tabular}{llll}
\hline & Min & Mean $\pm \mathrm{SD} / \mathrm{median}\left(\mathrm{P}_{25}-\mathrm{P}_{75}\right)$ & Max \\
\hline Leukocyte count & $1.1 \times 10^{9} / \mathrm{L}$ & $(17.6 \pm 11.8) \times 10^{9} / \mathrm{L}$ & $50.4 \times 10^{9} / \mathrm{L}$ \\
Neutrophils count & $0.2 \times 10^{9} / \mathrm{L}$ & $(14.0 \pm 10.8) \times 10^{9} / \mathrm{L}$ & $41.5 \times 10^{9} / \mathrm{L}$ \\
SCRP & 2.6 & $103(60-142) \mathrm{mg} / \mathrm{L}$ & $>180 \mathrm{mg} / \mathrm{L}$ \\
Procalcitonin $^{a}$ & 0.1 & $60(1.9-73.0) \mathrm{mg} / \mathrm{L}$ & $>200 \mathrm{mg} / \mathrm{L}$ \\
\hline
\end{tabular}

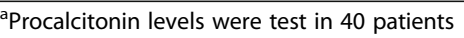

Treatment and outcome: The mean duration of treatment in these 66 patients was $16.3 \pm 13.2 \mathrm{~d}$ (range: $1-60$ days). The medium hospital stay was $17 \mathrm{~d}$ (p25-75: 623 days). Of the 66 patients, 25 (37.9\%) were admitted to pediatric intensive care unit (PICU) and the median ICU stay was 5 days. The details of the therapy in 66 patients were summarized in Table 2. Two (3.0\%) patients did not receive any antibiotics because of abandoning therapy or death within $2 \mathrm{~h}$ of admission. Fourteen (21.2\%) were treat with single antibiotic, and 50 (75.8\%) had been treated with 2 or more than 2 antibiotics, either in combination or sequentially. Eighteen (27.3\%) cases required surgical debridement and 18 (27.3\%) cases received intravenous immunoglobulin. Seventeen (25.8\%) patients received mechanical ventilation for a median duration of 3 days. Fifty-three (80.3\%) patients survived and $7(13.2 \%)$ of which had sequelae including $4(7.5 \%)$ with limp, $2(3.8 \%)$ with hemiplegia and 1 (1.9\%) with epilepsy. The total fatalities were 13 (19.7\%) cases, within which 7 (53.8\%) died in $24 \mathrm{~h}$ of admission and 10 (76.9\%) died in $72 \mathrm{~h}$ of admission. The case fatality ratio (CFR) was $53.3 \%$ in the case of STSS. Only 1 (1.5\%) patient who died had an underlying condition as cerebral palsy and was abandoned. Secondary transmission was

Table 4 Resistance of S. pyogenes strains to 10 antibiotics in 2011-2017

\begin{tabular}{llll}
\hline Antibiotics (N) & $\mathrm{S}(\mathrm{N}, \%)$ & $\mathrm{I}(\mathrm{N}, \%)$ & $\mathrm{R}(\mathrm{N}, \%)$ \\
\hline Penicillin G (63) & $63(100.0)$ & $0(0.0)$ & $0(0.0)$ \\
Ceftriaxone (36) & $36(100.0)$ & $0(0.0)$ & $0(0.0)$ \\
Cefotaxime (54) & $54(100.0)$ & $0(0.0)$ & $0(0.0)$ \\
Vancomycin (63) & $63(100.0)$ & $0(0.0)$ & $0(0.0)$ \\
Lynezolid (51) & $51(100.0)$ & $0(0.0)$ & $0(0.0)$ \\
Erythromycin (63) & $6(9.5)$ & $1(1.6)$ & $56(88.9)$ \\
Clindamycin (59) & $10(16.9)$ & $1(1.7)$ & $48(81.4)$ \\
Levofloxacin (58) & $55(94.8)$ & $2(3.5)$ & $1(1.7)$ \\
SXT (25) & $6(24.0)$ & $1(4.0)$ & $18(72.0)$ \\
Tetracycline (38) & $2(5.3)$ & $5(13.2)$ & $31(81.5)$ \\
\hline
\end{tabular}

$S$ sensitive, I intermediate, $R$ resistant, $S X T$ sulfamethoxazole-trimethoprim ${ }^{a}$ Drug-susceptibility test was not performed in three strains owing to the death of the three patients. Antibiotics tested in the nine hospitals differed during these years not found in this study. Hospital-patient disputes occurred in $6(9.1 \%)$ patients.

\section{Discussion}

ISPDs are uncommon but serious infections with high CFR. There are few publications on this subject at a regional level, particularly in the field of pediatrics. In the current study, only 66 pediatric patients were identified based on culture from 9 tertiary hospitals in 8 years. The low case number could be due to prior antibiotics usage that interfered with the etiological diagnosis of this disease. Because most of the patients were previously healthy children and the CFR was relative higher, hospital-patient disputes occurred in $9.1 \%$ of all patients, which caused huge trouble for the hospital. Thus, early recognition of this disease for effective treatment is really important. ISPDs have a broad and evolving clinical spectrum. Previous reports identified skin as a potential source for main trigger that leads to clinical manifestation of bacteremia or sepsis $[6,7]$, which were in accordance with our findings that $31.8 \%$ of the patients had skin or soft tissue infections as predisposing factors, indicating that pediatricians and emergency physicians must be aware of this possibility when treat skin or soft tissue infection. Zachariadou et al. [8] reported that varicella and streptococcal pharyngotonsillitis was the main predisposing factors in children, which was not supported in the present study. Neonates and infants $<1$ y are susceptible to S. pyogenes infections [5], and our study showed that $16.7 \%$ of all patients were neonates, which may be associated with their mother being a carrier or infected with this bacteria [9]. Most of the patients had a maximum temperature $>39.0^{\circ} \mathrm{C}$ and the fever tended to be ardent. High levels of leukocyte counts, CRP and procalcitonin concentration were found in most patients, which suggests that $S$. pyogenes infection is associated with relatively serious inflammation.

Determination of resistance to antibiotics in strains helps antibiotic choice in therapy. Even at present, penicillinnonsusceptible $S$. pyogenes are absence or extremely rare. Thus, penicillin remains the first-choice for treatment and is also a surrogate for ampicillin, amoxicillin, cefotaxime and ceftriaxone, in treating infectious disease caused by this bacterium. In the present study, all of the isolates were sensitive to beta-lactams. Most of the patients were cured with 
beta-lactams in therapy, which indicates the consistency of the antimicrobial activity of beta-lactams in vitro and in vivo. Macrolides are important alternatives for allergic patients and lincosamides are recommended together with beta-lactams in treating invasive infections [10,11]. For reasons that are not completely clear, macrolides-resistance is highly variable across countries. In Norway during 2010$2014,<4 \%$ of the included S. pyogenes were resistant to erythromycin [12]. Chochua et al. reported that of the 1454 invasive isolates, $12.7 \%$ were nonsusceptible to erythromycin [13]. During 2008-2013 in Finland, an increase of erythromycin resistance (1.9 to $8.7 \%)$ and clindamycin $(0.9$ to $9.2 \%$ ) were found [14]. Wajima et al. reported that $54.4 \%$ of the 283 invasive isolated were erythromycin-resistant [15]. In Asia, macrolides-resistance even higher. Lu et al. reported that 93.5 and $94.2 \%$ of the strains isolated from 2009 to 2016 were resistant to erythromycin and clindamycin, respectively [16]. Similarly, we found that 88.9 and $81.4 \%$ of the tested strains were resistant to erythromycin and clindamycin, which indicated that macrolides would not be the alternatives even for penicillin-allergic patients with $S$. pyogenes infection in China. The high macrolides-resistance of $S$. pyogenes isolates may associate with wide usage of macrolides in this population. Addition of a high dosage of clindamycin is recommended because of its excellent tissue penetration and improving the outcome by modulating virulence factors of clindamycinsusceptible and clindamycin-resistant $S$. pyogenes [11], In addition, clindamycin is an important adjunctive antibacterial, however, in the current study, most of the patients did not receive clindamycin, the possible explanation is that pediatrician choose antibiotics for therapy were based on antibiotic-resistance only and did not realize it's role on inhibiting virulence factors of the bacteria.

The onset and progression of ISPD can be rapid, and the associated mortality is high especially those complicated with STSS [17]. Given the rapid clinical progression, effective management of ISPD hinges on early recognition of the disease and prompt initiation of supportive care together with antibacterial therapy and early surgical debridement of infected tissue. Early institution of intravenous immunoglobulin therapy should be considered in cases of STSS and severe invasive infection, including necrotizing fasciitis $[9,11]$. However, only $27.3 \%$ of the patients received intravenous immunoglobulin, which may associate with the mortality in the present study was higher than in other study which were not more than $15 \%[2-4,7,18]$. In cases of severe invasive infections, it is often difficult to distinguish among bacterial infections before cultures become available and so antibiotics choice must include coverage of both of Gram-positive and Gram-negative bacteria. Rapid antigen detection tests [19] and PCR assay will help antibiotic prescriptions in the management of life-threatening $S$. pyogenes infections. Linder et al. [20] reported that compared with non-immunocompromised patients, immunocompromised patients are more likely to develop STSS and have a higher mortality, which were not identified in the present study.

There are limitations in this study. This study is retrospective based on database in China. The number of included cases with ISPD was small, thus, the results should be interpreted carefully. Usually, specific emm-genotypes are association with ISPD, for example, emm 1 are the leading cause of invasive disease worldwide $[8,12,19,21$, $22]$, However, genotypes of $S$. pyogenes were not performed in the study. Hereafter, a nationwide survey is required to clarify the epidemiology, risk factors, clinical and microbiological characteristics of $S$. pyogenes invasion disease in children in China. Ongoing surveillance is required in order to undertake appropriate control measures and gain a greater understanding of this disease.

\section{Conclusions}

Invasive S. pyogenes infections often developed from skin or soft tissue infection, fever was the major symptom, sepsis was the most frequent presentation and STSS was the main cause of death in Chinese children. Ongoing surveillance is required to gain a greater understanding of this disease in China.

\section{Abbreviations}

CFR: Case fatality rate; CRP: C-reactive protein; GAS: Group A streptococcus; iGAS: Invasive GAS infection; PCR: Polymerase chain reaction; PICU: Pediatric intensive care unit; S. pyogenes: Streptococcus pyogenes; SD: Standard deviation; STSS: Streptococcal toxic shock syndrome; SXT: Sulfamethoxazoletrimethoprim

\section{Acknowledgements}

We thank Dr. Ying-Jie Lu, Boston Children's Hospital, for critical reading of the manuscript. We thank the patients and their families of the study for their participation and the physicians who had diagnosed and treated the patients in our study.

\section{Funding}

Not applicable

\section{Availability of data and materials}

The datasets used and /or analyzed during the current study are available from the corresponding author on reasonable request.

\section{Authors' contributions}

HCZ conceived, initiated and designed the study, drafted the manuscript, prepared the study documents in the Children's Hospital, Zhejiang University School of Medicine, was leading investigator. YH coordinated the study, revised the manuscript critically for important intellectual content, prepared the study documents and enrolled the patients in Children's Hospital of Fudan University. XHM coordinated the study, revised the manuscript, prepared the study documents and enrolled the patients in Children's Hospital of Chongqing Medical College. YLH enrolled the patients and analyzed the data in Shanxi Children's Hospital. LAW enrolled the patients and analyzed the data in Qilu Children's Hospital of Shandong University. LQ enrolled the patients and analyzed the data in Ningbo Women and Children's Hospital. LHP enrolled the patients and analyzed the data in Taizhou Hospital. XZW enrolled the patients and analyzed the data in The Second Affiliated Hospital \&Yuying Children's Hospital of Wenzhou Medicial University. GW enrolled the patients and analyzed the data in Kaifeng 
Children's Hospital. CXJ enrolled the patients in the Children's Hospital, Zhejiang University School of Medicine, and revised the manuscript. WCO and JCM responsible for data management and performed the statistical analysis. All authors read and approved the final manuscript.

\section{Ethics approval and consent to participate}

This study was approved by the Ethics Committee and the Institutional Board of Privacy and Security at the hospitals (2018-IEC-047). It was performed under the institutions' opt-out passive consent policy. The Ethics Committee and the Institutional Board of Privacy and Security at the hospitals approved the waiver. Patients and guardians wishing to withdraw from the study were able to contact the principal investigator through information provided on notifications publicly posted by the institution's ethics committee.

\section{Consent for publication}

Not applicable

\section{Competing interests}

The authors declare that they have no competing interests.

\section{Publisher's Note}

Springer Nature remains neutral with regard to jurisdictional claims in published maps and institutional affiliations.

\section{Author details}

'Division of Infectious Diseases, The Children's Hospital, Zhejiang University School of Medicine, Hangzhou 310003, People's Republic of China. ${ }^{2}$ Division of Infectious Diseases, Children's Hospital of Fudan University, Shanghai 201102, People's Republic of China. ${ }^{3}$ Division of Infectious Diseases, Chongqing Medical University Affiliated Children's Hospital, Chongqing 400014, People's Republic of China. ${ }^{4}$ Department of Cardiology, Shanxi Children's Hospital, Taiyuan 030013, People's Republic of China. ${ }^{5}$ Division of Infectious Diseases, Qilu Children's Hospital of Shandong University, Jinan 250022, People's Republic of China. ${ }^{6}$ The Intensive Care Unit, Ningbo Women and Children's Hospital, Ningbo 315012, People's Republic of China. 'The intensive Care Unit, Taizhou Hospital of Zhejiang Province, Linhai 317000, People's Republic of China. ${ }^{8}$ Division of Infectious Diseases, The Second Affiliated Hospital \&Yuying Children's Hospital of Wenzhou Medicial University, Wenzhou 325027, People's Republic of China. ${ }^{9}$ Division of Infectious Diseases, Kaifeng Children's Hospital, Kaifeng 475000, People's Republic of China. ${ }^{10}$ Department of Clinical Laboratory, The Children's Hospital, Zhejiang University School of Medicine, Hangzhou 310003, People's Republic of China. " Department of Clinical Laboratory, Children's Hospital of Fudan University, Shanghai 201102 , People's Republic of China.

${ }^{12}$ Department of Clinical Laboratory, Chongqing Medical University Affiliated Children's Hospital, Chongqing 400014, People's Republic of China.

Received: 12 October 2018 Accepted: 14 May 2019

Published online: 05 June 2019

\section{References}

1. Ralph AP, Carapetis JR. Group a streptococcal diseases and their global burden. Curr Top Microbiol Immunol. 2013;368:1-27.

2. O'Loughlin RE, Roberson A, Cieslak PR, Lynfield R, Gershman K, Craig A, et al. The epidemiology of invasive group a streptococcal infection and potential vaccine implications: United States, 2000-2004. Clin Infect Dis. 2007;45(7): 853-62.

3. Tapiainen T, Launonen S, Renko M, Saxen H, Salo E, Korppi M, et al. Invasive group a streptococcal infections in children: a nationwide survey in Finland. Pediatr Infect Dis J. 2016;35(2):123-8.

4. Boyd R, Patel M, Currie BJ, Holt DC, Harris T, Krause V. High burden of invasive group a streptococcal disease in the Northern Territory of Australia. Epidemiol Infect. 2016;144(5):1018-27

5. Seale AC, Davies MR, Anampiu K, Morpeth SC, Nyongesa S, Mwarumba S, et al. Invasive group a streptococcus infection among children, rural Kenya. Emerg Infect Dis. 2016;22(2):224-32.

6. Cancellara AD, Melonari P, Firpo MV, Mónaco A, Ezcurra GC, Ruizf L, et al. Multicenter study on invasive Streptococcus pyogenes infections in children in Argentina. Arch Argent Pediatr. 2016;114(3):199-208.
7. Sivagnanam S, Zhou F, Lee AS, O'Sullivan MV. Epidemiology of invasive group a Streptococcus infections in Sydney, Australia. Pathology. 2015;47(4): 365-71.

8. Zachariadou L, Stathi A, Tassios PT, Pangalis A, Legakis NJ, Papaparaskevas J. Hellenic strep-euro study group. Differences in the epidemiology between paediatric and adult invasive Streptococcus pyogenes infections. Epidemiol Infect. 2014;142(3):512-9.

9. Yamada T, Yamada T, Yamamura MK, Katabami K, Hayakawa M, Tomaru U, et al. Invasive group a streptococcal infection in pregnancy. J Inf Secur. 2010;60(6):417-24

10. Andreoni F, Zürcher C, Tarnutzer A, Schilcher K, Neff A, Keller N, et al. Clindamycin affects group a streptococcus virulence factors and improves clinical outcome. J Infect Dis. 2017;215(2):269-77.

11. Steer AC, Lamagni T, Curtis N, Carapetis JR. Invasive group a streptococcal disease: epidemiology, pathogenesis and management. Drugs. 2012:72(9): 1213-27.

12. Naseer U, Steinbakk M, Blystad H, Caugant DA. Epidemiology of invasive group a streptococcal infections in Norway 2010-2014: a retrospective cohort study. Eur J Clin Microbiol Infect Dis. 2016;35(10):1639-48.

13. Chochua S, Metcalf BJ, Li Z, Rivers J, Mathis S, Jackson D, et al. Population and whole genome sequence based characterization of invasive group a streptococci recovered in the United States during 2015. MBio. 2017;19(8):5.

14. Smit PW, Lindholm L, Lyytikäinen O, Jalava J, Pätäri-Sampo A, Vuopio J. Epidemiology and emm types of invasive group a streptococcal infections in Finland, 2008-2013. Eur J Clin Microbiol Infect Dis. 2015;34(10):2131-6.

15. Wajima T, Morozumi M, Chiba N, Shouji M, Iwata S, Sakata H, et al. Associations of macrolide and fluoroquinolone resistance with molecular typing in Streptococcus pyogenes from invasive infections, 2010-2012. Int J Antimicrob Agents. 2013;42(5):447-9.

16. Lu B, Fang Y, Fan Y, Chen X, Wang J, Zeng J, et al. High prevalence of macrolide-resistance and molecular characterization of Streptococcus pyogenes isolates circulating in China from 2009 to 2016. Front Microbiol. 2017:8:1052.

17. Hua CZ, Yu H, Yang LH, Xu HM, Lyu Q, Lu HP, et al. Streptococcal toxic shock syndrome caused by Streptococcus pyogenes: a retrospective study of 15 pediatric cases. Zhonghua Er Ke Za Zhi. 2018;56(8):587-91 [Chinese].

18. Rudolph K, Bruce MG, Bruden D, Zulz T, Reasonover A, Hurlburt D, et al. Epidemiology of invasive group a streptococcal disease in Alaska, 2001 to 2013. J Clin Microbiol. 2016:54(1):134-41.

19. Gazzano V, Berger A, Benito Y, Freydiere AM, Tristan A, Boisset S, et al. Reassessment of the role of rapid antigen detection tests in diagnosis of invasive group a streptococcal infections. J Clin Microbiol. 2016:54(4):994-9.

20. Linder KA, Alkhouli L, Ramesh M, Alangaden GA, Kauffman CA, Miceli MH. Effect of underlying immune compromise on the manifestations and outcomes of group a streptococcal bacteremia. J Inf Secur. 2017;74(5):450-5.

21. Imöhl M, Fitzner C, Perniciaro S, van der Linden M. Epidemiology and distribution of 10 superantigens among invasive Streptococcus pyogenes disease in Germany from 2009 to 2014. PLoS One. 2017:12(7):e0180757.

22. Williamson DA, Morgan J, Hope V, Fraser JD, Moreland NJ, Proft T, et al. Increasing incidence of invasive group a streptococcus disease in New Zealand, 2002-2012: a national population-based study. J Inf Secur. 2015; 70(2):127-34.

Ready to submit your research? Choose BMC and benefit from:

- fast, convenient online submission

- thorough peer review by experienced researchers in your field

- rapid publication on acceptance

- support for research data, including large and complex data types

- gold Open Access which fosters wider collaboration and increased citations

- maximum visibility for your research: over $100 \mathrm{M}$ website views per year

At BMC, research is always in progress.

Learn more biomedcentral.com/submissions 